\title{
Evaluation of dynamic Parameters and developing calculation Methods of Parameters of pneumatic vibratory Mechanism of Bag Filters regeneration
}

\author{
Viktor Sopov ${ }^{*}$, Nikolay Emelianenko, Nikolay Balera, and Yuri Zhuravlov \\ Kharkiv National University of Civil Engineering and Architecture, Mechanical and technological \\ Faculty, Sumskaya str., 40, Kharkiv, 61002, Ukraine
}

\begin{abstract}
A scheme of membrane pneumatic mechanism of shaking bag filter for cleaning the air from dust is developed. The research findings of dynamics of air mechanic vibratory bag filter system are shown. The concept of revised methods for calculating main parameters of membrane pneumatic vibratory mechanism of the bag filter regeneration is developed.
\end{abstract}

\section{Introduction}

Many modern industrial production processes are accompanied with the formation of air disperse systems from airborne solid particles of dust. An efficient equipment to capture fine particles up to 5 micron is bag filters [1-3].

Bag filters are used in metallurgical, chemical, cement and other industries. Efficiency of dust collection in the bag filter depends on the size of particles, characteristics of the filter matter, hydraulic resistance and the way of regeneration [2].

Regeneration of the filter matter is carried out by mechanical bags shaking, pulsed jet or a combination of these methods [2-5]. Mechanical shaking of the filters is performed by [3]:

- rapid swinging from side to side without changing the tension of the filter elements made from non-resistant to bend fabrics;

- method of undulations of filter elements made from elastic and thick fabrics;

- fabric tension undulating change in vertical bag filters when lifting and lowering the bag.

Bag filters are a popular type of product. For example LLC "Eco filter" [6] produces bag filters for new generation of air output of $1000 \mathrm{~m}^{3} / \mathrm{h}$ to $2000000 \mathrm{~m}^{3} / \mathrm{h}$, which are used in the production of dry mixes, cement, building materials, metallurgy and so on. Bag filter RFU10 with bags regeneration by vibration shaking produced by the company permits to clean the dust-laden air to a residual impurity concentration of $15 \div 20 \mathrm{mg} / \mathrm{m}^{3}$. The filter has a built-in mechanism for bags regeneration by periodical vibration.

Bag filter schematic diagrams with shaking mechanism driven by the electromechanical vibrators or cam mechanisms are shown in [1-6]. The method of mechanical shaking allows

\footnotetext{
Corresponding author: vsopov@ukr.net
} 
cleaning filter elements from dust without stopping the system and automates the process of the air dusting.

Records of this article are environmentally oriented and dedicated to improving bag filters by application to them an air vibratory drive of a bag shaking mechanism.

The purpose of the research - assessment of dynamic parameters and development of the methods for calculating parameters of the proposed design of the pneumatic vibratory mechanism of the bag filter regeneration.

\section{Structure and operation principle of filter with pneumatic vibratory mechanism of bags fabric regeneration}

Construction schematic diagram of the filter with a pneumatic vibratory mechanism of bags fabric regeneration [7] is shown in Fig. 1.

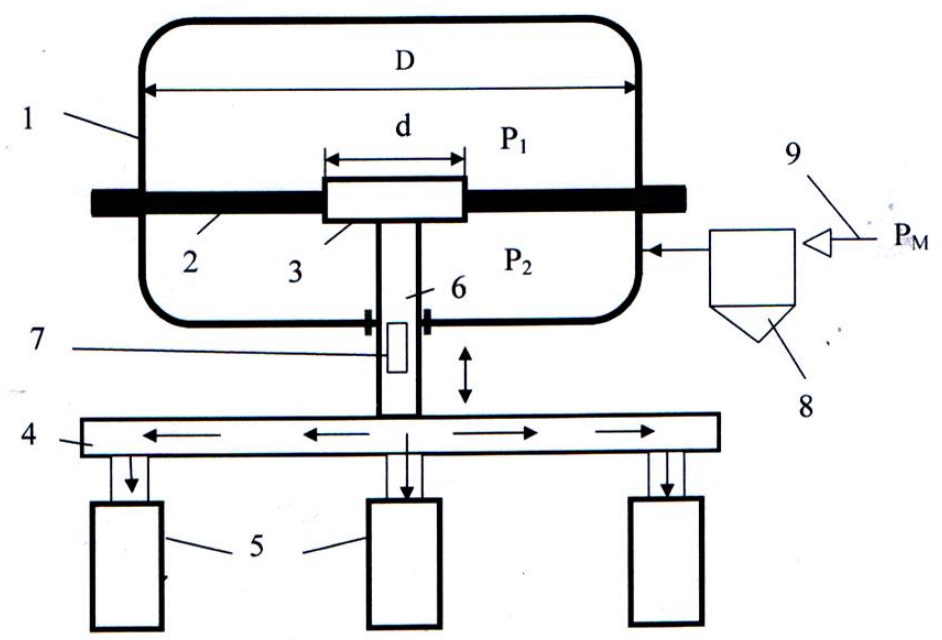

Fig. 1. Schematic diagram of the filter with a pneumatic vibratory mechanism of bags fabric regeneration.

The filter consists of a membrane 2 installed in a base 1 with a solid center 3 connected with a crosspiece 4 . The membrane 2 divides the base 1 into the upper and bottom planar surface and joined to the crosspiece 4 with bags 6,5 by using a hollow stem with a hole 7 . The lower cage of the base 1 is connected with the air supply line 9 through the electric valve 8 .

Before the work starts the upper cage of the base 1 is filled with compressed air under the pressure that is substantially lower than the main line pressure.

When operating the compressed air flows to the lower chamber of the base 1 from the line 9 through the valve 8 , acts on the membrane 2 which flexes upward, moves the rod 6 and the frame 4 with bags 5 upward until the hole 7 connects the lower chamber with cages of the rod 6 and the frame 4; herewith the compressed gas flows to the bags 2 and further blows them. Then the pressure in the lower chamber decreases, and under its own weight and the resilient force of the membrane 2 and the compressed air in the upper chamber of the rod 6 , the frame 4 and bags 5 move down with an additional acceleration which intensifies the bag filter fabric cleaning process. After exiting the holes 7 of the rod 6 out of the lower chamber gas pressure increases and the membrane 2 once again raises the rod with a frame and filters upward, causing vibrations of the movable filter system. 


\section{Elements of dynamics of filter with pneumatic vibratory mechanism of bags fabric regeneration}

Basic theory, advantages and methods of calculation of the pneumatic vibratory drives are set out in the work of E.V. Hertz [8].

The equation of motion of traverse with filter bags in a vertical direction for the vibration mode, taking into account the research findings [9-11] is presented in the form:

$$
M \frac{d^{2} x}{d t^{2}}+b \frac{d x}{d t}+c x=\Delta P \cdot(1-k) \cdot S_{e f f} \cdot \cos (2 \pi v t)
$$

where $\mathrm{M}$ - the mass of vibrating parts of the system (traverse, bags, rod and solid center); $b$ - coefficient of resistance to the working body vibrations; $\mathrm{c}$ - reduced factor of the membrane tension, compressed air and bags suspender; $\Delta \mathrm{P}=\mathrm{P}_{2}-\mathrm{P}_{1}$ - pressure difference in chambers; Seff - effective membrane area:

$$
S_{\text {eff }}=\frac{\pi}{12} \cdot\left(D^{2}+D \cdot d+d^{2}\right)
$$

where D - inner diameter of the base 1; $\mathrm{d}$ - washer diameter (solid center 3); $\mathrm{k}$ - coefficient of reduction of the pressure fall in the pneumatic cages taking into account the chamber volume $\mathrm{V}$ and vibration frequency (determined by the method [11]).

A particular solution of equation (1) is obtained in the form of:

$$
x=A \cdot \cos (\omega t-\phi),
$$

traverse move amplitude; $\varphi$ - phase shift angle:

$$
\begin{aligned}
& A=\frac{\Delta P \cdot S_{e f f} \cdot(1-k)}{\sqrt{\left(c-M \omega^{2}\right)^{2}+c M \cdot\left(\frac{\psi \cdot \omega}{2 \pi}\right)^{2}}} \\
& \phi=\operatorname{arctg}\left(\frac{\sqrt{c M} \cdot \psi \cdot \omega}{2 \pi \cdot\left(c-M \omega^{2}\right)}\right)
\end{aligned}
$$

$\omega=2 \pi \nu$ - vibration angular frequency; $\psi$ - coefficient of energy dissipation.

\section{Calculation methods of basic parameters of pneumatic vibratory drive of filter cleaning mechanism}

The effective area of the membrane from (4) is:

- outer diameter of the membrane working part $D=\sqrt{\frac{12 S_{e f f}}{\pi\left(1+\lambda+\lambda^{2}\right)}}$;

- metal washer diameter (solid center) $d=\lambda \cdot D$.

Air flow in pneumatic vibratory drive:

$$
Q=\frac{\beta \cdot P_{M}}{P_{A}} \cdot S_{e f f} \cdot\left(H+A_{n}\right) \cdot v,
$$

where $\beta$ - coefficient of main line pressure reduction; $H$ - lower chamber height in a rest state of system; $\mathrm{P}_{\mathrm{M}}, \mathrm{P}_{\mathrm{A}}$ - the pressure of airline and atmosphere.

The diameter of the pipes and area of venting: 


$$
d_{\square}=\sqrt{\frac{4 \cdot V}{\pi \cdot u}} ; \quad f_{\text {vent }} \geq 0.25 \pi \cdot d_{\square}^{2},
$$

where $\mathrm{u}$ - average speed of the compressed air flow through the pipeline.

\section{Implementation of parameters calculation algorithm of bag filter with pneumatic vibratory drive of bags dedusting mechanism. Research analysis}

For parameters values of the filter with pneumatic vibratory drive of bags dedusting mechanism:

$$
\begin{aligned}
& \Delta P=0,20 ; 0,25 ; 0,30 \mathrm{MPa} ; \mathrm{M}=800 \mathrm{~kg} ; \\
& V=0,01 \mathrm{M}^{3} ; \quad \mathrm{c}=200000 \mathrm{~N} / \mathrm{m} ; \quad \mathrm{A}=0,02 \mathrm{~m}
\end{aligned}
$$

Diagrams shown in Fig. 2 are to define the required effective membrane area for different combinations of traverse with bags vibration frequency and pressure difference in cages.

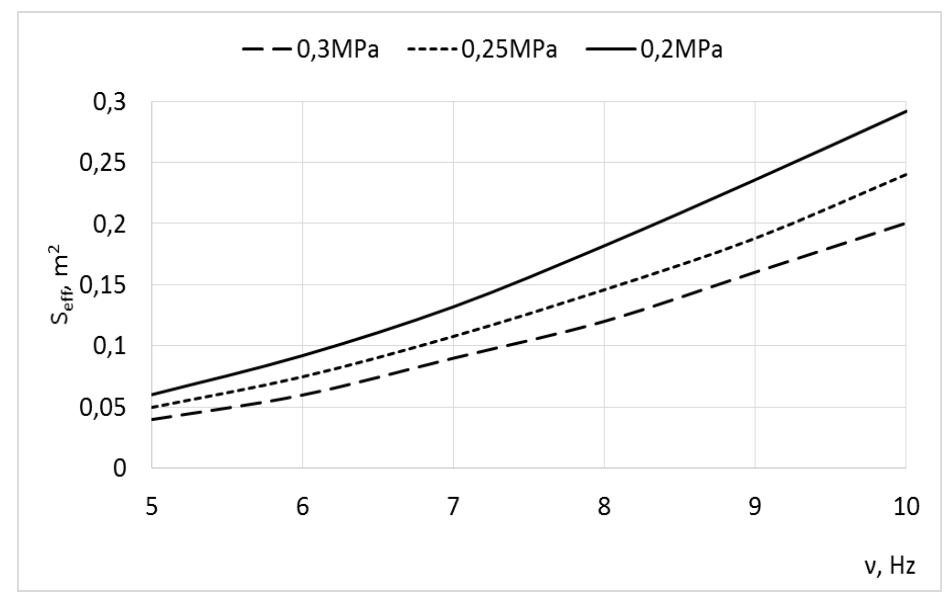

Fig. 2. Correspondence of the required effective membrane area of pneumatic vibratory mechanism to the bags shaking frequency and pressure difference in cages.

Diagrams shown in Fig. 3 are to determine the amplitude of oscillation depending on the system mass at a fixed pressure difference of the compressed air in cages $\Delta \mathrm{P}=0,2 \mathrm{MPa}$ for frequencies $v=5,8,10 \mathrm{~Hz}$. 


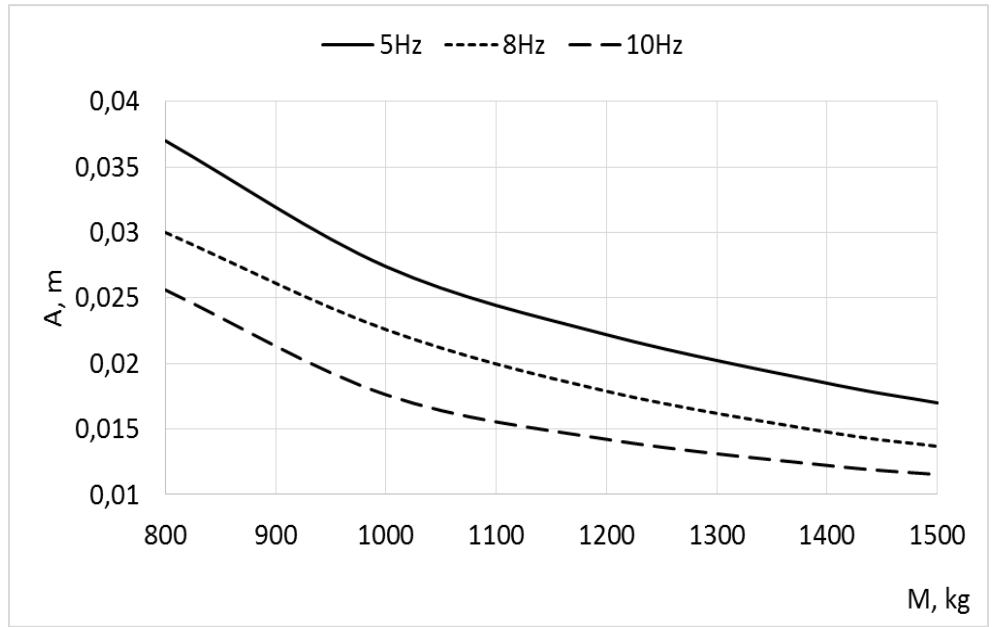

Fig. 3. Determination of traverse with bags amplitude of oscillations depending on the system mass at a fixed pressure difference in the cages of pneumatic vibratory drive $\Delta \mathrm{P}=0,2 \mathrm{MPa}$ for frequencies $\mathrm{v}=5$, $8,10 \mathrm{~Hz}$.

As you can see in Fig. 2 and 3, the maintaining the oscillation amplitude of traverse with bags on the same level as the frequency increases is achieved by the following actions:

- increasing the effective membrane area (with a constant pressure difference in chambers);

- increasing the pressure difference in chambers.

Diagrams shown in Fig. 4 and 5 are to determine the amplitudes of A displacements and accelerations of a traverse with bags at smooth change of the movable elements mass of the filter with pneumatic vibratory drive of bags dedusting mechanism within the range of $\mathrm{M}=800 \ldots 1400 \mathrm{~kg}$, shaking frequency $v=5 \mathrm{~Hz}$ and discrete change of the pressure difference in chambers $\Delta \mathrm{P}=0,1 ; 0,2 ; 0,3 \mathrm{MPa}$.

Proceeding from Fig. 4 to achieve stable amplitude of oscillations of the system at a constant frequency of shaking bags and increasing mass of movable parts of the filter the air pressure difference in the pneumatic cages should be enhanced. For example, increasing mass from 800 to $1400 \mathrm{~kg}$ while maintaining the amplitude $\mathrm{A}=0,02 \mathrm{~m}$, it is necessary to move to $\Delta \mathrm{P}=0,2 \mathrm{MPa}$ from the pressure difference $\Delta \mathrm{P}=0,1 \mathrm{MPa}$ because another way increasing the effective membrane area - should be used only at the bag filters design stage. At a constant vibrating parts mass of the filter, in the case of technological necessity to implement the increasing amplitude can be achieved by increasing the pressure difference.

Assuming the acceleration level sufficient to separate cement dust from the bags fabric technically realizable values of the amplitude and vibration frequency by a pneumatic mechanism of shaking bags can be determined. Moreover, for a given weight of vibrating parts of the system, including the traverse with bags the working values of the pressure difference in the base cages of the pneumatic mechanism and an effective membrane area must be assigned in accordance with the concept of the revised calculation methods that presented. 


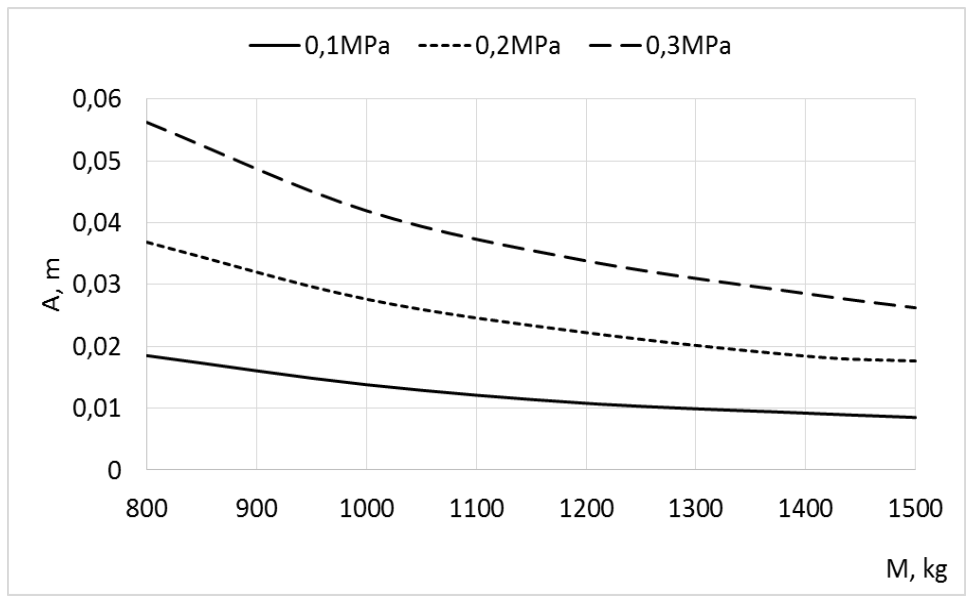

Fig. 4. Amplitude correspondence of the A traverse with bags displacement to mass $M$ of movable parts of the filter for different values of pressure difference in the drive cages of bags dedusting mechanism.

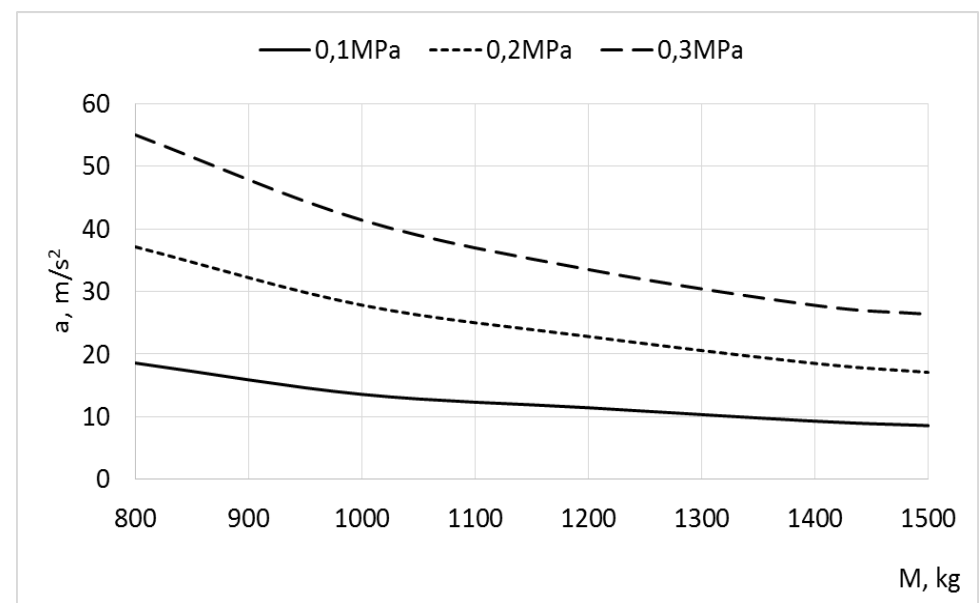

Fig. 5. Amplitudes acceleration determination of the filter vibratory system when changing mass of the filter movable parts $\mathrm{M}$ for different values of the pressure difference in the mechanism drive cages.

\section{Conclusions}

1. The proposed version of the pneumatic mechanism of filter bags shaking for cleaning the air from cement dust increases the technological opportunities, in particular at the expense of control of vibration parameters (frequency, amplitude, shaking force).

2. Correspondence obtained from the research allows estimating the dynamic characteristics of pneumatic vibratory drive of the filter bags shaking mechanism for cleaning the air from cement dust.

3. Obtained parameters calculation methods can be used to determine parameters of the membrane pneumatic vibratory drive of filter matter regeneration mechanism in the design of the bag filter.

The authors wish to thank A.S. Riabovol for her assistance in preparing this paper. 


\section{References}

1. M. Ja. Sapozhnikov, Mechanical equipment of enterprises of building materials, products and constructions (Higher School, Moscow, 1971)

2. G. Salewski, World Cement, 11 (2008)

3. A. G. Vetoshkin, Processes and devices of dust cleaning. (Penza State University Press, Penza, 2005)

4. L. V. Chekalov, Ecotechnika. Protection of atmospheric air of dust emissions, aerosols and mists, (Rus, Yaroslavl, 2004)

5. Bases of calculation of machinery and equipment of enterprises of building materials and products, Ed. V.S. Bogdanov, (TNT, Stary Oskol, 2013)

6. Yun-Haeng Joe, Joonmok Shim, and Hyun-Seol Park, Powder Technology, 321, (2017).

7. Ukraine Patent UA 85918 C2 Bag Filter. Balera M. D., Emelianenko M. G., Sopov V.P, No a 200705988; 10.03.2009, Bulletin 5, (2009)

8. E. V. Hertz, Dynamics of Pneumatic machines, (Mashinostroenie, Moscow, 1985)

9. N. G. Emelianenko, International symposium of construction machines. Technical University "Otto von Guericke", Magdeburg, (1987)

10. N. G. Emelianenko, Bulletin National technical university "Kharkiv politechnical institute", 30, (2006)

11. N. G. Emelianenko, Scientific Bulletin of Civil Engineering, 42, (2007) 